\title{
Multi-Parameter Dominant Grouping of Discontinuities in Rock Mass Using Improved ISODATA Algorithm
}

\author{
Qing Ding $\left(\mathbb{D},{ }^{1}\right.$ Runqiu Huang, ${ }^{2}$ Fengyan Wang $\left(\mathbb{D},{ }^{1,2}\right.$ Jianping Chen $(\mathbb{D}){ }^{3}$ \\ Mingchang Wang, ${ }^{1}$ and Xuqing Zhang ${ }^{1}$ \\ ${ }^{1}$ College of Geo-Exploration Science and Technology, Jilin University, Changchun 130026, China \\ ${ }^{2}$ State Key Laboratory of Geohazard Prevention and Geoenvironment Protection, Chengdu 610059, China \\ ${ }^{3}$ College of Construction Engineering, Jilin University, Changchun 130026, China \\ Correspondence should be addressed to Fengyan Wang; wangfy@jlu.edu.cn
}

Received 23 January 2018; Revised 15 June 2018; Accepted 27 November 2018; Published 12 December 2018

Academic Editor: Alessandro Formisano

Copyright (C) 2018 Qing Ding et al. This is an open access article distributed under the Creative Commons Attribution License, which permits unrestricted use, distribution, and reproduction in any medium, provided the original work is properly cited.

\begin{abstract}
The dominant grouping of the discontinuities in a rock mass is crucial to investigating the rock structure and stability. The traditional grouping methods are mostly based on the discontinuity orientation. However, other discontinuity parameters nonnegligibly impact the properties of a rock mass. If two discontinuities have the same orientation but differ otherwise, their mechanical and hydraulic properties would differ. In the present study, orientation, trace length, opening degree, and undulation of discontinuities were used to develop a multi-parameter dominant discontinuity grouping method that utilizes an improved ISODATA algorithm. The developed method uses several indicators, such as the weighted Euclidean distance and standard deviation, to iteratively update the clustering centers of the discontinuities. A clustering validity index was introduced for assessment and optimization of the discontinuity grouping results, and an adaptive grouping model that considers the weight of each grouping parameter was developed. By grouping discontinuities generated by Monte Carlo stochastic simulation, defects existing in the grouping based on the orientation only were illustrated, and the rationality of the established adaptive grouping model was verified. The engineering practicability of the method was further verified by using it to group discontinuities measured in the Dongsheng Quarry in Jingyuetan, Changchun, China.
\end{abstract}

\section{Introduction}

Through the different stages of its development and existence, a rock mass undergoes complex geological processes, resulting in the formation of a large number of discontinuities of various sizes and types within the rock. Compared with the rock type, the orientation, density, and size of the discontinuities have greater effects on the mechanical properties of the rock mass $[1,2]$. The measurement and statistical analysis of the discontinuity parameters are thus important to the study of the mechanical properties of a rock mass and related engineering stability assessments [3].

The original method for grouping discontinuities involves the use of the pole isosceles and joint rose map to describe the output state of the discontinuities, after which the discontinuity groups are identified by manual analysis. The grouping achieved by this method is subjective to some extent owing to its dependence on the experience and professional knowledge of the analyst. Shanley [4] and Mahtab [5] pioneered the combination of mathematical methods with the dominant grouping of discontinuities. However, it was more difficult to use this method to determine the radius of the sphere when searching for the density point. Nevertheless, Chen Jianping et al. [6] improved this method and introduced the right-hand rule, which utilizes principles of probability and statistics. Hammah et al. [7] proposed a discontinuity grouping method based on a fuzzy $\mathrm{C}$-means algorithm. The method, however, had the shortcoming of being only applicable to local search optimization. R. Jimenez [8] integrated the fuzzy K-means method and the spectral method to study the grouping of discontinuities and indicated the degree of the membership for each discontinuity to its assigned 
clustering center by the color scale. $\mathrm{Xu}$ et al. [9] proposed a fuzzy C-means cluster analysis method based on the mutative scale chaos optimization algorithm, which overcame the drawbacks of the traditional fuzzy C-means algorithm that can only be used for local optimization. Yan et al. [10] proposed an improved K-means algorithm based on particle swarm optimization, which reduced the sensitivity of grouping results to the initial clustering center and achieved a global optimization in the grouping of discontinuities. Liu et al. [11] realized the grouping of discontinuities based on a modified affinity propagation algorithm, which solved the difficulty of initial clustering center selection during grouping. Zhan et al. [12] used a full sphere to represent orientation data based on a finite mixture model to achieve clustering of discontinuities with high dip angles. In addition to the grouping methods mentioned above, different global optimization algorithms for discontinuity grouping have been proposed [13-17].

The above discontinuity grouping methods are only based on the discontinuity orientation. However, in practice, discontinuities with the same orientation but differing in other parameters would exhibit differing mechanical properties. It is also inappropriate to group discontinuities based on the dip and dip angle for engineering purposes, owing to the inadequate reliability of the grouping result [18]. Tokhmechi [19] highlighted the shortcomings of grouping discontinuities based on only their orientation and the necessity of considering multiple grouping indexes. Zhou et al. [20] used orientation, spacing, and roughness as the parameters for grouping discontinuities. Four different methods were used to achieve the grouping of discontinuities, namely, the nearest neighbor, $\mathrm{K}$-means, fuzzy $\mathrm{C}$-means, and vector quantization. Behzad Tokhmechi [19] used a variety of methods, including rose diagrams and $\mathrm{K}$-means and grouped the discontinuities with the orientation, filling material, and filling percentage as the grouping parameters to study the shortcomings of common clustering methods. Xu et al. [21] proposed a method of grouping discontinuities that utilized a mutative scale chaos optimization algorithm. Discontinuities were grouped using the orientation, length, width, and surface morphology as grouping parameters. Song et al. [22] proposed a method based on the quantum particle swarm optimization algorithm, considering the orientation, trace length, opening degree, and surface morphology of discontinuities and realized multi-parameter grouping of discontinuities. The above grouping methods have their respective advantages, shortcomings, and applicability. For example, the method that utilizes a mutative scale chaos optimization algorithm has the disadvantages of a long operation time, low efficiency, and repeated calculation. Based on a finite mixture model, the grouping method that uses a full sphere to represent orientation is more suitable for discontinuities with high dip angles. Compared with the traditional clustering algorithm, the ISODATA algorithm can automatically adjust according to the intermediate results in the iterative process of the algorithm and has better adaptability.

In addition to the orientation, trace length is another important parameter for describing the quality of rock mass; opening degree and undulation also have certain influence on rock mass quality. This paper proposes an improved

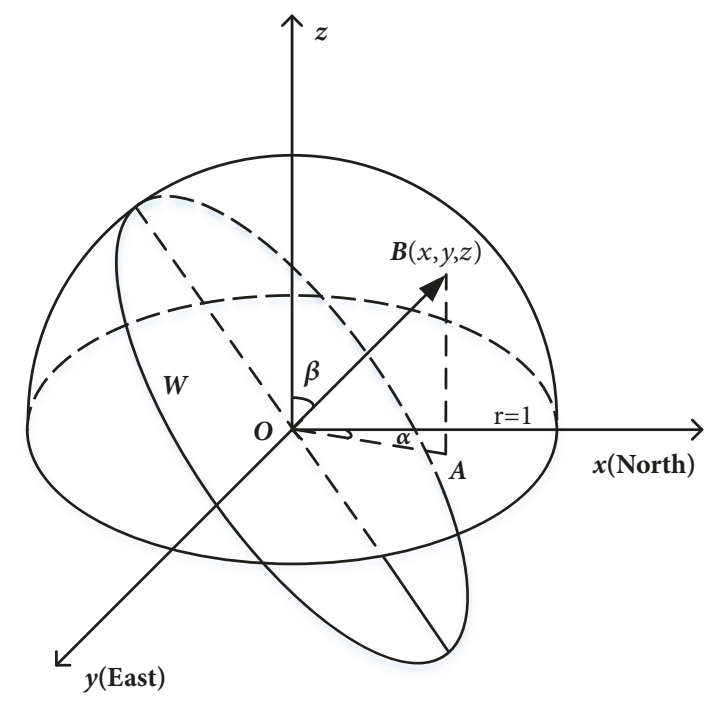

FIgURE 1: Representation of the orientation.

discontinuity grouping method that utilizes the ISODATA algorithm [23]. The method considers four grouping parameters, namely, orientation, trace length, opening degree, and undulation. To achieve dominant grouping of the discontinuities, the proposed method also introduces several indicators of the grouping parameters, namely, the weighted Euclidean distance [24], standard deviation, and clustering validity. The results of a grouping test conducted using simulation data verified the necessity of grouping with multiple parameters and the effectiveness of the method. The proposed grouping method was further applied to the discontinuities in a slope at the Dongsheng Quarry, Jingyuetan, Changchun, China, and the clustering validity index was used to assess the results. The optimal grouping of the discontinuities was also obtained, affording effective reference for rock stability assessment and disaster prevention.

\section{Methods and Materials}

\subsection{Model for Multi-Parameter Grouping of \\ Discontinuities Using Improved ISODATA Algorithm}

2.1.1. Expression of Discontinuity Grouping Parameters. An improved ISODATA algorithm was used for the discontinuities grouping in this study. The employed grouping parameters were the orientation (dip and dip angle), trace length, opening degree, and undulation. The first three parameters could be calculated by digital close-range photogrammetry and quantitatively described, while the undulation could only be described qualitatively based on the shape of the exposed part of the discontinuity.

In the processing and analysis of the orientation of a discontinuity, it is often assumed that the discontinuity occurs in a plane in space [25], and the unit normal vector of the plane is used to represent the orientation.

In the Cartesian coordinate system in Figure 1, the positive north direction is the positive $x$ direction, the positive east direction is the positive $y$ direction, and $\alpha \beta$, respectively, 
TABLE 1: Quantification of undulation.

\begin{tabular}{lc}
\hline Undulation & Numeric value \\
\hline Straight & 1 \\
Wavy & 2 \\
Zigzag & 3 \\
\hline
\end{tabular}

represent the dip and dip angle of the discontinuity. $\overrightarrow{O B}$ is the unit normal vector corresponding to the discontinuity $W$ and is expressed as

$$
\overrightarrow{O B}=(x, y, z)=(\cos \alpha \sin \beta, \sin \alpha \sin \beta, \cos \beta)
$$

Although the undulation of a discontinuity can only be qualitatively described based on the morphological characteristics of the exposed part of the rock mass, it is necessary to introduce a representative and specific numerical value into the algorithm used for discontinuity grouping. The specific quantification method used in this study is presented in Table 1.

With the quantification of the undulation, the grouping parameters of a discontinuity $W$ can be expressed as $W=$ $(\alpha, \beta, T L, e, S)$, where $T L$ is the trace length, $e$ is the opening degree, and $S$ is the undulation.

Discontinuity is the geological interface formed and developed continuously in the rock mass during the long historical process of rock formation and geological action. The orientation, trace length, opening degree, and undulation are four important parameters describing different properties of discontinuity. According to the extension length, the depth of the cutting, the width of the fracture zone, and the mechanical effects, the discontinuity can be divided into five levels, such as faults, unconformities, layers, joint cracks, and the like. Different levels of discontinuities have different forms and sizes, which make the parameters (such as orientation, trace length, opening degree, and undulation) indicating their properties different. In addition, rock mass is a heterogeneous and discontinuous complex medium; the parameters of different discontinuities at the same level are different. Furthermore, the unit and numerical range of different parameters of the same discontinuity are also different.

Owing to the significantly differing ranges of the grouping parameters, they are normalized to reduce the magnitude discrepancies before introduction into the grouping algorithm. The unit normal vector of the discontinuity is adopted as the normalized form of the orientation, while the normalized forms of the other parameters are given by

$$
\begin{aligned}
T L_{i}^{\prime} & =\frac{T L_{i}-T L_{\min }}{T L_{\text {max }}-T L_{\min }} \quad(i=1,2,3, \ldots, N) \\
e_{i}^{\prime} & =\frac{e_{i}-e_{\min }}{e_{\max }-e_{\min }} \quad(i=1,2,3, \ldots, N) \\
S_{i}^{\prime} & =\frac{S_{i}-S_{\min }}{S_{\text {max }}-S_{\min }} \quad(i=1,2,3, \ldots, N)
\end{aligned}
$$

where $T L_{i}, e_{i}$, and $S_{i}$, respectively, denote the trace length, opening degree, and undulation of the $i$-th discontinuity;
$T L_{\text {max }}$ and $T L_{\text {min }}$, respectively, denote the maximum and minimum trace lengths among all the discontinuities; $e_{\max }$ and $e_{\text {min }}$, respectively, denote the maximum and minimum opening degrees among all the discontinuities; $S_{\max }$ and $S_{\min }$, respectively, denote the maximum and minimum undulation among all the discontinuities; and $N$ is the total number of discontinuities. The normalized grouping indexes of discontinuities are used for the discontinuity grouping: $W=$ $\left(x, y, z, T L^{\prime}, e^{\prime}, S^{\prime}\right)$.

\subsubsection{Improved Iterative Self-Organizational Discontinuities} Grouping Model. The ISODATA algorithm is more frequently used for data clustering analysis. Compared with the traditional grouping algorithm, the ISODATA algorithm is capable of making automatic adjustments based on the results obtained midway into an operation process. This enables human-computer interaction and produces better final grouping results [26] through adaptive grouping.

Based on the ISODATA algorithm, the authors introduced the weighted Euclidean distance for attaching weights to the different grouping indexes, thus according them differing importance in the grouping process. In using this improved ISODATA algorithm for discontinuity grouping, it is necessary to select a certain number of initial clustering centers. In the present study, two discontinuities were selected based on the principle of the maximum Euclidean distance and introduced into the algorithm as the initial clustering centers; the Euclidean distance was expressed as follows:

$$
D\left(W_{i}, W_{j}\right)=\left[\sum_{z=1}^{n}\left(r_{z}\left(W_{i}^{z}-W_{j}^{z}\right)^{2}\right)\right]^{1 / 2}
$$

where $D\left(W_{i}, W_{j}\right)$ denotes the Euclidean distance between discontinuities $i$ and $j, n$ is the number of grouping indexes, $r_{z}$ is the weight of the $z$-th grouping index of the discontinuities, and $W_{i}^{z}$ denotes the $z$-th grouping index of the discontinuity $i$.

After normalizing the raw data of the discontinuities and setting the initial clustering centers, they are introduced into the improved ISODATA algorithm for processing. Figure 2 shows the main flowchart of the process.

Based on the Euclidean distance between discontinuities and the more forms of fuzzy math in fuzzy C-means (FCM) [27], the memberships of the discontinuities in the clustering centers can be calculated using

$$
g_{i j}^{(k)}=\left[\frac{\left(1 / D^{2}\left(W_{j}, U_{i}^{(k)}\right)\right)}{\sum_{i=1}^{N(k)}\left(1 / D^{2}\left(W_{j}, U_{i}^{(k)}\right)\right)}\right]^{1 / m-1}
$$

where $g_{i j}^{(k)}$ denotes the membership degree of discontinuity $j$ in clustering center $i$ in the $k$-th iteration, $U_{i}^{(k)}$ denotes clustering center $i$ in the $k$-th iteration, $N(k)$ is the number of clustering centers in the $k$-th iteration, and $m$ is the weighted index.

The clustering centers are updated based on the degree of membership of the discontinuities in the clustering centers during the iterations. After an update, it is necessary to 


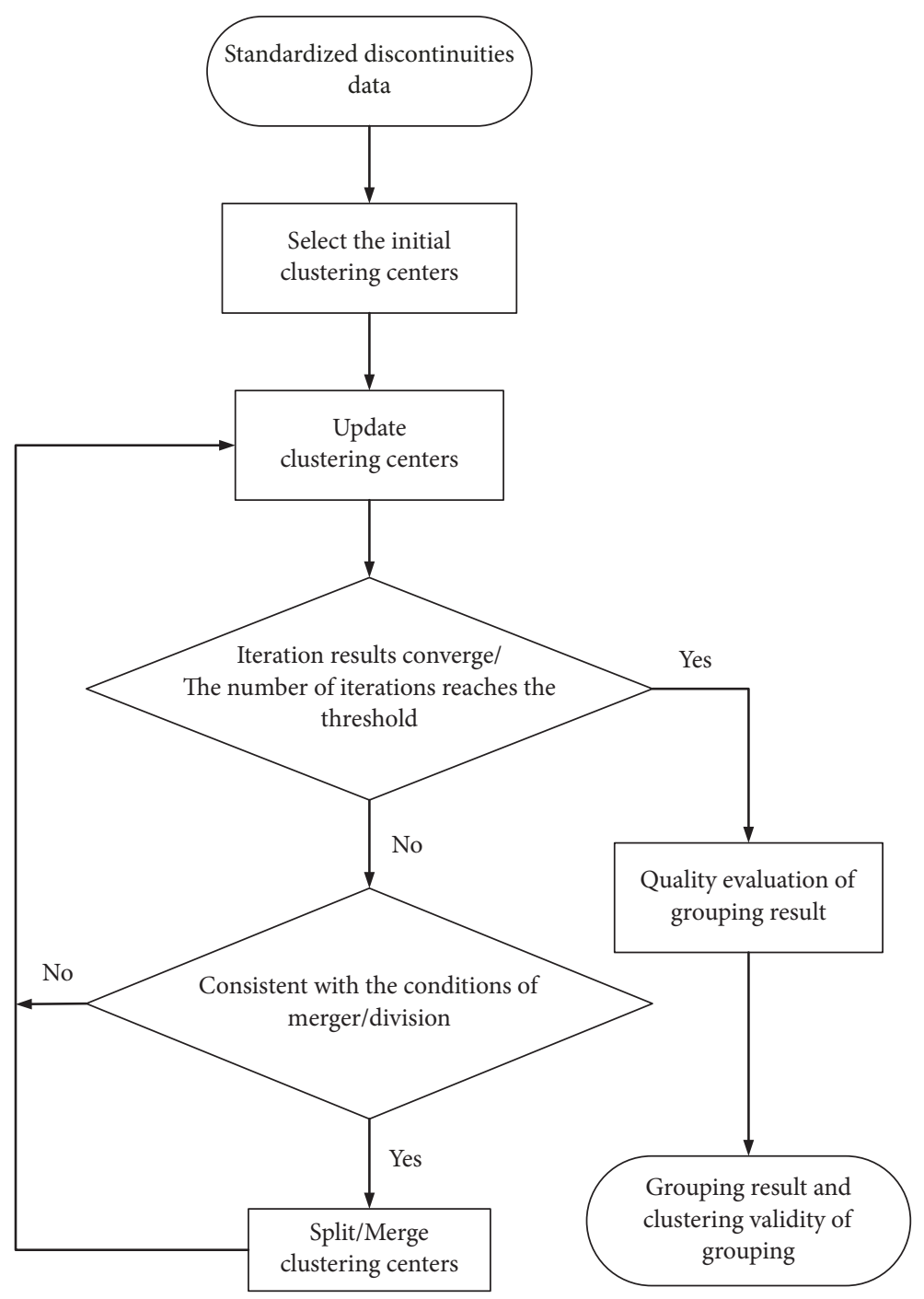

FIGURE 2: Flowchart of the discontinuity grouping model.

reprocess the unit vector that represents the orientation to ensure that the final results can be inversely normalized.

$$
\begin{aligned}
& \widehat{U}_{i}=\frac{\sum_{j=1}^{N}\left(g_{i j}\right)^{m} W_{j}}{\sum_{j=1}^{N}\left(g_{i j}\right)^{m}} \\
& x^{\prime}=\frac{x}{\left(x^{2}+y^{2}+z^{2}\right)^{1 / 2}} \\
& y^{\prime}=\frac{y}{\left(x^{2}+y^{2}+z^{2}\right)^{1 / 2}} \\
& z^{\prime}=\frac{z}{\left(x^{2}+y^{2}+z^{2}\right)^{1 / 2}}
\end{aligned}
$$

In (7), $\widehat{U}_{i}$ is the updated clustering center $i$. In (8), $x^{\prime}, y^{\prime}$, and $z^{\prime}$ are the unit vectors representing the discontinuity orientation data that are normalized again after the clustering centers are updated.
After the clustering centers are updated, the iteration results are assessed for convergence. The occurrence of convergence indicates that comparison of the results of the $n$-th iteration of the algorithm with those of the $(n-1)$ th and $(n-2)$ th iterations, respectively, reveals that the differences are less than the threshold. That is, the algorithm does not split and merge the clustering centers in the $(n-2)$ th and $(n-1)$ th iterations and ends the algorithm and outputs the results at the $n$-th iteration. The algorithm also sets an exit by fixing the number of iterations, when the number of iterations exceeds the set threshold, the algorithm is terminated, and the results are outputted. If the iterative results do not converge and the number of iterations does not reach the set threshold, it is determined whether the clustering centers meet the splitting or merging conditions.

When the number of iterations of the algorithm is odd and the Euclidean distance between two clustering centers is smaller than the set threshold, the merging conditions of the clustering centers are satisfied. A new clustering 
center is then generated by obtaining the weighted sum of the two clustering centers based on the weighted indexes and memberships. Only the two clustering centers with the smallest Euclidean distance are combined in each iteration.

$$
\widehat{U}_{p q}=\frac{\sum_{j=1}^{N}\left(W_{j}\left(g_{p j}\right)^{m}+W_{j}\left(g_{q j}\right)^{m}\right)}{\sum_{j=1}^{N}\left(\left(g_{p j}\right)^{m}+\left(g_{q j}\right)^{m}\right)}
$$

In (9), clustering centers $p$ and $q$ are merged into a new clustering center $\widehat{U}_{p q}$.

When the number of iterations of the algorithm is even and the standard deviation of any grouping indexes of any clustering center is greater than the set threshold, the splitting condition of the clustering center is satisfied. In each iteration, the clustering center that contains the grouping indexes with the largest standard deviation performs the splitting process. With the other indicators of the clustering center remaining unchanged, the grouping indicator with a standard deviation greater than the threshold splits by one-half of the standard deviation to generate two new clustering centers.

$$
\sigma_{l}^{z}=\sqrt{r_{z} \frac{1}{N} \sum_{j=1}^{N}\left(W_{j}^{z}-U_{l}^{z}\right)^{2}\left(g_{l j}\right)^{m}} ; \quad z=1,2, \ldots, n
$$

In (10), $\sigma_{l}^{z}$ is the standard deviation of the grouping indicator of the $z$-th discontinuity in clustering center 1 in the iterative process.

If the clustering center numbered 1 satisfies the splitting condition, the standard deviation of the grouping indicator $i$ in this iteration is greater than the threshold, and it is the maximum value of the standard deviation of each clustering index of all clustering centers. The clustering center is split into two new clustering centers according to the following equation.

$$
\begin{aligned}
& W_{l}^{i}(1)=W_{l}^{i}+\frac{\sigma_{l}^{i}}{2} \\
& W_{l}^{j}(1)=W_{l}^{j}, \quad j=1,2, \ldots, n, j \neq i \\
& W_{l}^{i}(2)=W_{l}^{i}-\frac{\sigma_{l}^{i}}{2} \\
& W_{l}^{j}(2)=W_{l}^{j}, \quad j=1,2, \ldots, n, j \neq i
\end{aligned}
$$

In (11), $W_{l}^{i}$ denotes a grouping indicator numbered $\mathrm{i}$ that needs to be split in the original cluster center 1 and $W_{l}^{j}$ denotes the remaining grouping indicator that does not require splitting in the original cluster center $1 . W_{l}^{i}(1), W_{l}^{i}(2)$, $W_{l}^{j}(1)$, and $W_{l}^{j}(2)$ are the corresponding grouping indicators in the two new clustering centers generated after the original clustering center 1 splits.

After the completion of the algorithm iteration, the obtained final grouping index needs to be inversely normalized and outputted. The antinormalization formulas of the dip and dip angle are presented as (12) and (13), while those of the other indicators are presented as (14), (15), and (16).

$$
\begin{aligned}
\alpha & = \begin{cases}0^{\circ} & x \geq 0, y=0 \\
180^{\circ} & x<0, y=0 \\
180^{\circ} \times \frac{\arccos (x)}{\pi} & y>0 \\
360^{\circ}-180^{\circ} \times \frac{\arccos (x)}{\pi} & y<0\end{cases} \\
\beta & =180^{\circ} \times \frac{\arccos (z)}{\pi} \\
T L & =T L^{\prime}\left(T L_{\text {max }}-T L_{\text {min }}\right)+T L_{\text {min }} \\
e & =e^{\prime}\left(e_{\text {max }}-e_{\text {min }}\right)+e_{\text {min }}
\end{aligned}
$$

The results of the discontinuity grouping vary with the weight setting of each grouping parameter. An index of the clustering effectiveness was thus introduced as a measure of the merits of the grouping results with respect to the weighting configuration [28]. Known as the Xie-Beni index $v_{X B}$, it is one of the measures used to assess the validity of the grouping results. When the grouping results are good, the data of the internal discontinuities of each group are more concentrated, and the distances between different groups are larger. The smaller the value of $v_{X B}$, the better the results of the discontinuity grouping. The equation of this clustering validity index is as follows:

$$
v_{X B}=\frac{\sum_{i=1}^{C} \sum_{j=1}^{N} g_{i j}^{2} D\left(W_{j}, U_{i}\right)}{N\left(\min _{i \neq k} D\left(U_{i}, U_{k}\right)\right)}
$$

where $C$ is the number of clustering centers in the final grouping results and $\min _{i \neq k} D\left(U_{i}, U_{k}\right)$ is the minimum Euclidean distance between clustering centers in the final results.

\subsection{Experimental Data}

2.2.1. Simulation Data. Using the Monte Carlo stochastic simulation method, 200 discontinuities were generated based on the four above-mentioned discontinuity parameters (see Table 2) [25]. By comparing the results obtained by the present method with the known centers of discontinuities and analyzing the size of the differences to test the reasonability of the method, the data of the discontinuities generated by the computer simulation included the dip and dip angle, trace length, opening degree, and undulation. The trace length data was normally distributed; that of the dip and dip angle had a bivariate normal distribution; and that of the opening degree was evenly distributed. The undulation values were directly allotted based on the groupings. The pole map generated from the simulated data is shown in Figure 3.

From the pole diagram, it can be clearly seen that the discontinuities are divided into two groups with large differences. Grouping of discontinuities on the basis of orientation also verified the intuitive judgment from the polar map, 
TABLE 2: Parameters of the simulation discontinuities.

\begin{tabular}{|c|c|c|c|c|c|c|c|c|c|c|c|c|c|}
\hline \multirow{3}{*}{ Group } & \multicolumn{6}{|c|}{ Bivariate normal distribution } & \multirow{2}{*}{\multicolumn{3}{|c|}{$\begin{array}{l}\text { Normal distribution } \\
\text { Trace length }(\mathrm{m})\end{array}$}} & \multirow{2}{*}{\multicolumn{2}{|c|}{$\begin{array}{l}\text { Uniform Distribution } \\
\text { Opening degree }(\mathrm{mm})\end{array}$}} & \multirow{3}{*}{ Undulation } & \multirow{3}{*}{ Numbe } \\
\hline & \multicolumn{3}{|c|}{$\operatorname{Dip}\left({ }^{\circ}\right)$} & \multicolumn{3}{|c|}{ Dip angle $\left({ }^{\circ}\right)$} & & & & & & & \\
\hline & Mean & Range & Var & Mean & Range & Var & Mean & Range & Var & Mean & Range & & \\
\hline 1 & 242.9 & $212 \sim 269$ & 170.6 & 44.8 & $26 \sim 62$ & 63.3 & 2.85 & $2.35 \sim 3.51$ & 0.09 & 1.00 & $0.80 \sim 1.20$ & 1.0 & 40 \\
\hline 2 & 284.4 & $253 \sim 316$ & 209.2 & 59.6 & $41 \sim 80$ & 54.3 & 4.68 & $3.70 \sim 5.47$ & 0.19 & 2.60 & $2.41 \sim 2.79$ & 3.0 & 60 \\
\hline 3 & 41.0 & $3 \sim 71$ & 153.3 & 74.5 & $61 \sim 82$ & 30.3 & 1.44 & $0.89 \sim 2.02$ & 0.04 & 0.68 & $0.51 \sim 0.80$ & 1.0 & 60 \\
\hline 4 & 73.3 & $41 \sim 104$ & 185.4 & 69.5 & $57 \sim 88$ & 43.4 & 3.55 & $2.94 \sim 4.17$ & 0.09 & 2.02 & $1.81 \sim 2.17$ & 2.0 & 40 \\
\hline
\end{tabular}

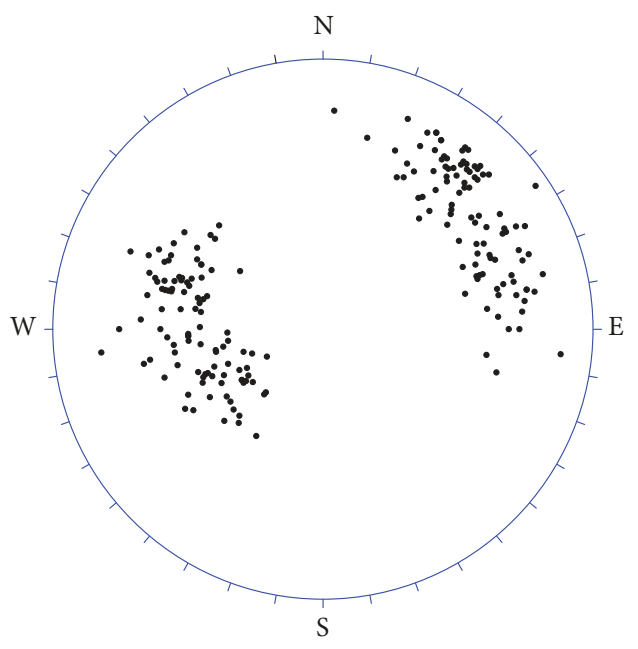

Figure 3: Pole map of simulation data.

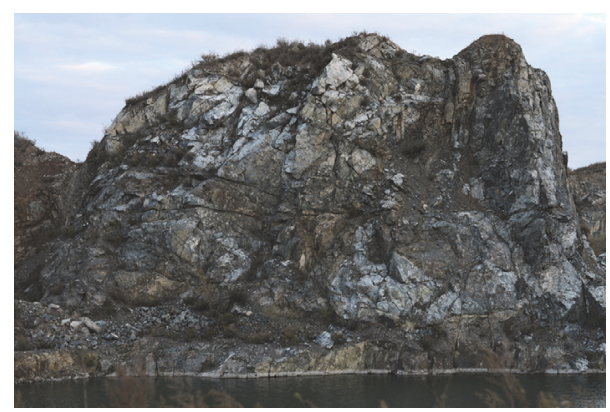

FIgURE 4: Actual situation of the study slope.

which is totally different from the case where the simulated data are divided into four groups. Therefore, if only orientation is considered, the grouping result of discontinuities is flawed. This paper proposes a multi-parameter dominant grouping of discontinuities in rock mass using an improved ISODATA algorithm, considering orientation, trace length, opening degree, and undulation. According to the measurement accuracy and importance of each parameter, different weights are assigned to achieve a comprehensive analysis and judgment of discontinuities and more accurate grouping results are obtained.

2.2.2. Measured Data. Dongsheng Quarry is located in Dongsheng Village within the Jingyue Tourism Economic

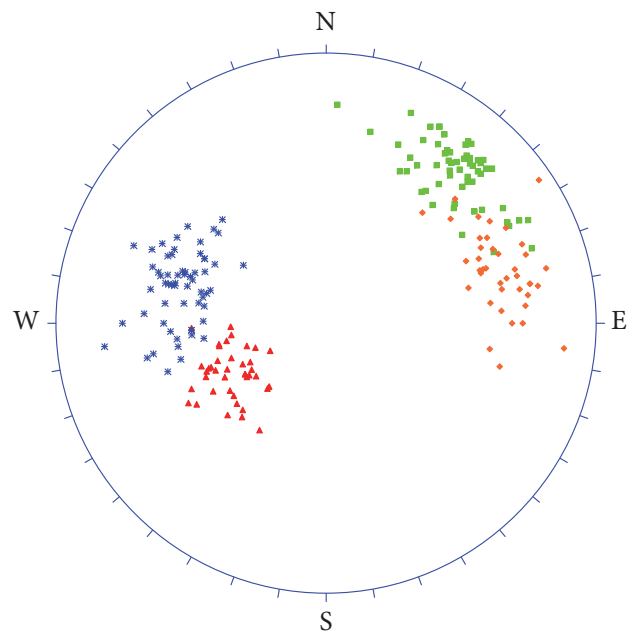

FIGURE 5: Pole map of the grouping results of the discontinuity simulation data. Note: the colors and shapes of the plot points correspond to the different groups as indicated in Table 4.

Development Zone, Changchun City, Jilin Province. The geographical coordinates of the quarry are $125^{\circ} 30^{\prime} \mathrm{E}$ and $43^{\circ} 48^{\prime} \mathrm{N}$. The landscape of the quarry mainly consists of hilly landforms. A typical slope located in the same statistical homogeneous area (see Figure 4) was selected for the present study. The lithology of the slope rock mass is andesite and discontinuities develop evenly.

The slope, which is about $50 \mathrm{~m}$ long and $30 \mathrm{~m}$ high, is relatively unaffected by erosion and weathering, with the discontinuities quite intact. A total of 683 discontinuity data units were acquired from the slope by digital closerange photogrammetry, as detailed in Table 3 [29], and used to calculate and analyze the engineering example of the slope to verify the engineering practicability of the proposed method.

\section{Results}

3.1. Rationality Test of the Method. The grouping results of the simulation discontinuity data were compared with the known discontinuity grouping, as presented in Table 4 . Figure 5 shows the pole map of the discontinuity grouping results, while Figure 6 shows the density maps of the poles generated by the discontinuities of each group. The clustering validity indicator $v_{X B}$ of the grouping results was determined to be 0.0605 . 


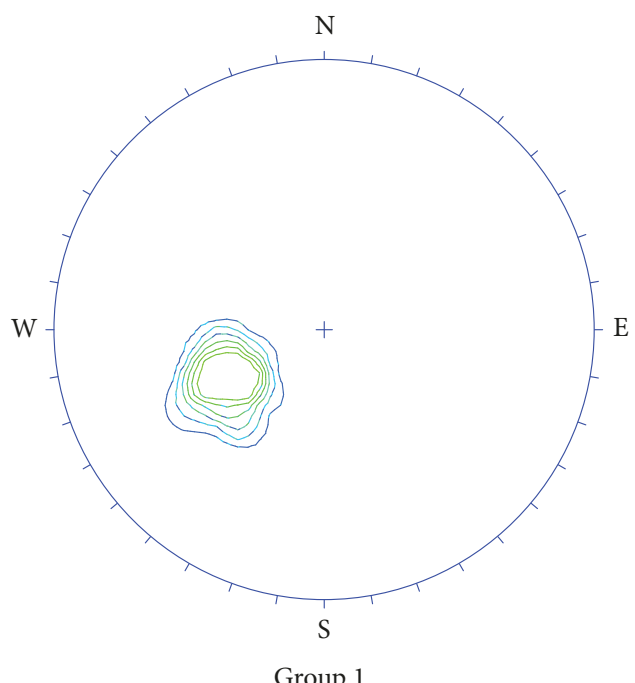

Group 1

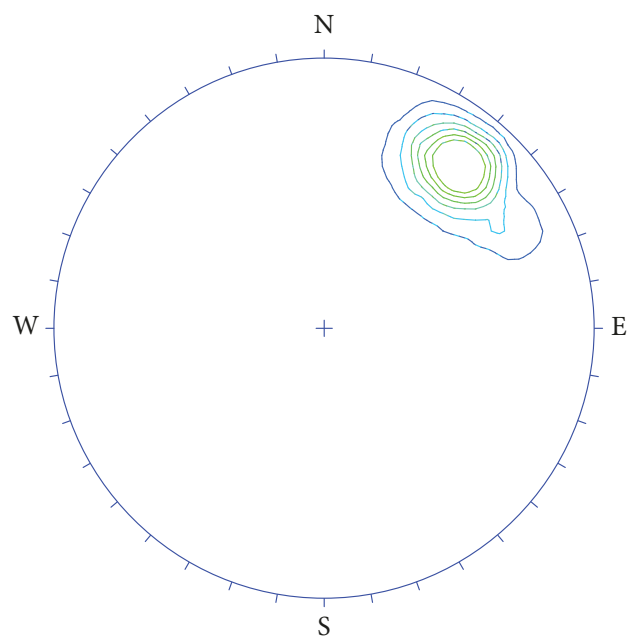

Group 3

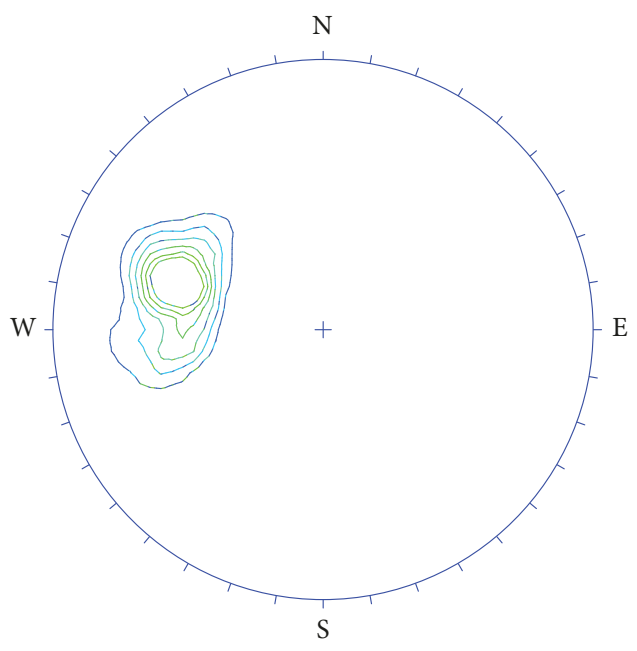

Group 2

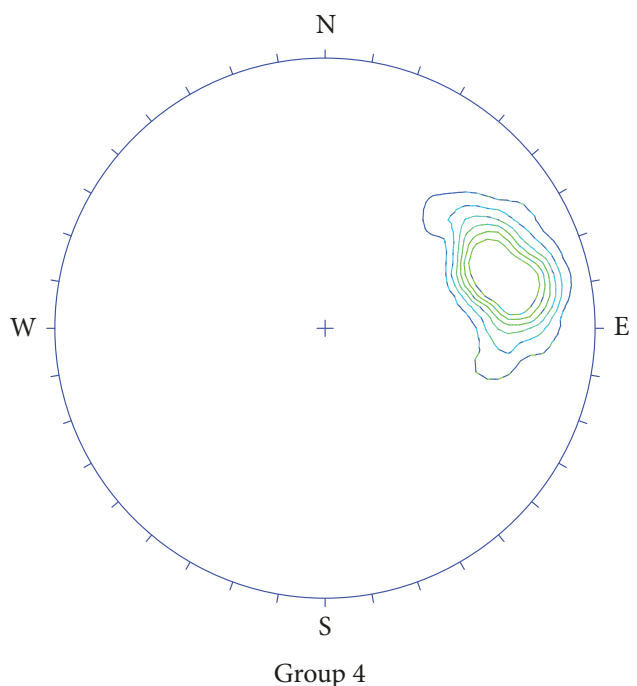

FIgURE 6: Polar ISO density graph.

TABLE 3: Discontinuity data acquired from the study slope.

\begin{tabular}{|c|c|c|c|c|c|}
\hline $\begin{array}{l}\text { Discontinuity } \\
\text { number }\end{array}$ & $\operatorname{Dip}\left({ }^{\circ}\right)$ & Dip angle $\left({ }^{\circ}\right)$ & Trace length(m) & Opening degree $(\mathrm{mm})$ & Undulation \\
\hline 1 & 22.52 & 52.30 & 2.96 & 50 & 1 \\
\hline 2 & 22.71 & 68.85 & 0.98 & 0 & 1 \\
\hline 3 & 358.89 & 58.73 & 1.73 & 0 & 1 \\
\hline 4 & 124.43 & 87.86 & 0.62 & 0 & 1 \\
\hline$\vdots$ & $\vdots$ & $\vdots$ & $\vdots$ & $\vdots$ & $\vdots$ \\
\hline 681 & 26.57 & 87.96 & 1.28 & 0 & 1 \\
\hline 682 & 8.74 & 60.64 & 1.28 & 20 & 2 \\
\hline 683 & 186.7 & 58.89 & 2.4 & 20 & 1 \\
\hline
\end{tabular}

3.2. Analysis of the Engineering Application. The mechanical strength of the slope discontinuities was observed to be relatively weak. Given that the slope discontinuities determine the anisotropy, inhomogeneity, and discontinuity of a rock mass [30], an investigation of the development and distribution of the slope discontinuities facilitates an evaluation of the stability of the rock mass. The grouping of the slope discontinuity data acquired from the Dongsheng Quarry in the present study was used as reference for further slope stability evaluation. 
TABLE 4: Comparison of known centers and grouping results.

\begin{tabular}{|c|c|c|c|c|c|c|c|}
\hline Group number & Category & $\operatorname{Dip}\left({ }^{\circ}\right)$ & Dip angle $\left({ }^{\circ}\right)$ & Trace length $(\mathrm{m})$ & Opening degree $(\mathrm{mm})$ & Undulation & Number \\
\hline \multirow{2}{*}{ Set $1(\boldsymbol{\Delta})$} & Known center & 242.9 & 44.8 & 2.85 & 1.00 & 1.0 & 40 \\
\hline & Calculation result & 251.6 & 44.1 & 2.88 & 1.01 & 1.0 & 40 \\
\hline \multirow{2}{*}{ Set $2(*)$} & Known center & 284.4 & 59.6 & 4.68 & 2.60 & 3.0 & 60 \\
\hline & Calculation result & 283.0 & 58.7 & 4.68 & 2.60 & 3.0 & 60 \\
\hline \multirow{2}{*}{ Set $3(\square)$} & Known center & 41.0 & 74.5 & 1.44 & 0.68 & 1.0 & 60 \\
\hline & Calculation result & 42.6 & 74.4 & 1.46 & 0.69 & 1.0 & 60 \\
\hline \multirow{2}{*}{ Set $4(\diamond)$} & Known center & 73.3 & 69.5 & 3.55 & 2.02 & 2.0 & 40 \\
\hline & Calculation result & 75.2 & 69.2 & 3.51 & 1.99 & 2.0 & 40 \\
\hline
\end{tabular}

TABLE 5: Clustering validity with respect to the grouping parameter weights.

\begin{tabular}{lccccc}
\hline Weight of orientation & Weight of trace length & Weight of opening degree & Weight of undulation & Number of groups & Validity indicator \\
\hline $1 / 4$ & $1 / 4$ & $1 / 4$ & $1 / 4$ & 3 & 0.3079 \\
$2 / 5$ & $3 / 10$ & $3 / 20$ & $3 / 20$ & 4 & 4 \\
$1 / 2$ & $1 / 4$ & $1 / 8$ & $1 / 8$ & 5 & 0.2523 \\
$3 / 5$ & $1 / 5$ & $1 / 10$ & $3 / 40$ & 5 & 0.2473 \\
$7 / 10$ & $3 / 20$ & $3 / 40$ & &
\end{tabular}

During the grouping process, the thresholds for the clustering center splitting and merging were all set to 0.1 . The orientation and the trace length are accorded more importance than opening degree, and undulation, and their measurement accuracy is also better than the latter. Therefore, they should be given more weight to make the grouping results more reasonable. The weight of the orientation in the grouping of the measured data ranged between $2 / 5$ and $7 / 10$, the weight of trace length was $1 / 2$ of the remaining weight, and the weight of opening degree and undulation was taken as $1 / 2$ of the weight of trace length. That is, the weight of orientation is the highest, followed by the weight of the trace length, and the weights of opening degree and undulation are the smallest. In this case, the orientation and trace length play a leading role in grouping discontinuities, and, at the same time, the influence of the opening degree and undulation on the grouping result is not ignored. In addition, the subdivision weights of each grouping indicator are also considered; that is, the weight of each indicator is $1 / 4$. The results of the clustering validation for different weights of the grouping indicators are presented in Table 5.

When the discontinuities of different rock masses are grouped, the minimum value of the clustering validity is taken as the allocation principle for grouping parameter weights. For the experimental slope, it can be observed from Table 5 that the validity index of the clustering is the lowest (0.2473) when the weight of orientation is $5 / 10$, the weight of the trace length is $1 / 4$, and the weights of both the opening degree and the weight of undulation both are $1 / 8$, and the grouping results of the discontinuities of the slope rock mass are optimal. For this weight configuration, the algorithm iterates 80 times and the results are stable and optimal. The 683 discontinuities are divided into four groups, as detailed in Table 6.

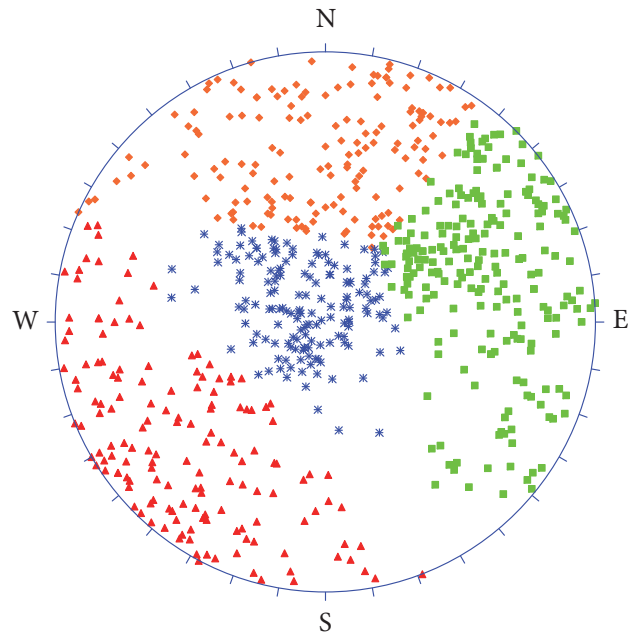

Figure 7: Pole map of the grouping results of the measured discontinuities. Note: the colors and shapes of the plot points correspond to the different groups as indicated in Table 6 .

The discontinuity grouping results are presented more intuitively and clearly in the pole map shown in Figure 7.

\section{Discussions}

It can be seen from Table 4 that there are only little differences between the known discontinuity grouping and the grouping results of the simulation discontinuity data. In addition, the clustering validity indicator $v_{X B}$ is small, verifying the reasonability of the proposed method for the dominant grouping of multi-parameter discontinuities. The grouping results were further processed to obtain a pole map of the 
TABLE 6: Best grouping of the measured discontinuity data.

\begin{tabular}{|c|c|c|c|c|c|c|}
\hline Group number & $\operatorname{Dip}\left({ }^{\circ}\right)$ & Dip angle $\left({ }^{\circ}\right)$ & Trace length $(\mathrm{m})$ & Opening degree $(\mathrm{mm})$ & Undulation & Number \\
\hline Set $1(\boldsymbol{\Delta})$ & 229.6 & 74.1 & 2.00 & 16.6 & 1.16 & 153 \\
\hline Set $2(*)$ & 276.2 & 10.8 & 2.37 & 11.3 & 1.22 & 157 \\
\hline Set $3(\square)$ & 72.3 & 65.7 & 2.22 & 14.6 & 1.10 & 216 \\
\hline Set $4(\diamond)$ & 25.2 & 64.9 & 2.18 & 8.5 & 1.26 & 157 \\
\hline
\end{tabular}

discontinuity orientations and polar ISO density graphs of the different discontinuity groups (Figures 5 and 6). The simulation discontinuities were ideally divided into four groups with clear boundaries, demonstrating the reliability of the grouping method.

The discontinuity data acquired from a slope in the Dongsheng Quarry were also grouped. It can be seen from Table 6 that the discontinuities of groups 1, 3, and 4 have large dip angles, indicating relatively high steepness. The discontinuities of group 2 have smaller dip angles, indicating relatively gentle slopes. In addition, the dip angles, trace lengths, and undulations of the discontinuities of groups 3 and 4 are similar, whereas their dips and opening degrees significantly differ. This informed the division of the discontinuities into two separate groups. The undulations of the discontinuities in all four groups are about 1 , indicating a relatively smooth overall slope.

It can be further seen from Figure 7 that the 683 discontinuities are divided into four groups with obvious boundaries. This confirms the correctness of the grouping results and the engineering practicability of the proposed discontinuity grouping method.

\section{Conclusions}

Although the traditional method for grouping discontinuities based on only their orientation is relatively simple, it has some drawbacks. When two discontinuities have the same orientation, they may exhibit different mechanical properties owing to their other parameters being different. This has some effects on the stability evaluation of the rock mass. Thus, a multi-parameter discontinuity grouping method that utilizes an improved ISODATA algorithm was proposed in this study. The proposed method is adaptable and can evaluate and optimize the grouping results. Following is a summary of the experimental implementation of the proposed method and the conclusions drawn from the study.

(1) Monte Carlo stochastic simulation was used to generate four sets of discontinuities data with a certain degree of discrimination. From the pole map generated by the simulated discontinuities, discontinuities are clearly divided into two groups. The grouping results based only on the orientation only the intuitive judgment from the pole map, which does not meet the actual situation. This not only shows that there are certain defects in the grouping of discontinuities based only on the orientation, but also shows the necessity of using multiple parameters to group discontinuities. The results of the multi-parameter grouping of the discontinuities using the improved ISODATA algorithm was compared with the known grouping to verify the rationality of the proposed method.

(2) Through close-range photogrammetry, discontinuities were acquired from a slope in the Dongsheng Quarry in Jingyue, Changchun City. This method of data acquisition has higher efficiency and better accuracy and can describe the three-dimensional information of the rock mass discontinuities more comprehensively. Through the method proposed in this paper, the acquired discontinuities were grouped. A clustering effectiveness index was introduced through experimental analysis for the comparison of different grouping results obtained using different grouping parameters with differing weights. Under the principle of the minimum clustering validity index, the optimal weight configuration was determined to consist of a weight of $1 / 2$ for orientation, $1 / 4$ for trace length, and $1 / 8$ for each opening degree and undulation. This weight configuration produced the best grouping results of the discontinuities of the slope rock mass in the present study, and a pole map of the orientation verified the correctness of the results. This confirmed the engineering practicability of the proposed method.

(3) Owing to the establishment of two different means of terminating the algorithm, it exhibits a higher computational efficiency compared with traditional methods, with the produced grouping affording important reference for rock stability assessment.

\section{Data Availability}

The simulated data and measured data used to support the findings of this study may be released upon application to the author named Fengyan Wang, who can be contacted by email: wangfy@jlu.edu.cn.

\section{Conflicts of Interest}

The authors declare that there are no conflicts of interest regarding the publication of this paper.

\section{Acknowledgments}

This work was supported by the National Natural Science Foundation of China (Grant nos. 41472243 and 41330636), the Opening Fund of State Key Laboratory of Geohazard Prevention and Geoenvironment Protection of China (Grant no. SKLGP2013K018), and the Open Fund of Key Laboratory of Urban Land Resources Monitoring and Simulation, Ministry of Land and Resources (Grant no. KF-2018-03-020). 


\section{References}

[1] T.-J. Song, J.-P. Chen, W. Zhang, L.-J. Xiang, and J.-H. Yang, "A method for multivariate parameter dominant partitioning of discontinuities of rock mass based on artificial bee colony algorithm," Rock and Soil Mechanics, vol. 36, no. 03, pp. 861-868, 2015.

[2] P. Lopes and M. Lana, "Analytical method for calculating the volume of rock blocks using available mapping data field," Mathematical Geosciences, vol. 49, no. 2, pp. 217-229, 2017.

[3] Y. Fadakar Alghalandis, P. A. Dowd, and C. Xu, "The RANSAC Method for Generating Fracture Networks from Micro-seismic Event Data," Mathematical Geosciences, vol. 45, no. 2, pp. 207224, 2013.

[4] R. J. Shanley and M. A. Mahtab, "Delineation and analysis of clusters in orientation data," Journal of the International Association for Mathematical Geology, vol. 8, no. 1, pp. 9-23, 1976.

[5] M. A. Mahtab and T. M. Yegulalp, "A rejection criterion for definition of clusters in orientation data," in Proceedings of the 22nd Symposium on Rock Mechanics, pp. 116-123, American Institute of Mining Metallurgy and Petroleum Engineers, New York, 1982.

[6] J.-P. Chen, B.-F. Shi, and Q. Wang, "Study on the dominant orientations of random fractures of fractured rock masses," Chinese Journal of Rock Mechanics and Engineering, vol. 24, no. 2, pp. 241-245, 2005.

[7] R. E. Hammah and J. H. Curran, "Fuzzy cluster algorithm for the automatic identification of joint sets," International Journal of Rock Mechanics and Mining Sciences, vol. 35, no. 7, pp. 889905, 1998.

[8] R. Jimenez, "Fuzzy spectral clustering for identification of rock discontinuity sets," Rock Mechanics and Rock Engineering, vol. 41, no. 6, pp. 929-939, 2008.

[9] L. M. Xu, J. P. Chen, Q. Wang, and F. J. Zhou, "Fuzzy C-means cluster analysis based on mutative scale chaos optimization algorithm for the grouping of discontinuity sets," Rock Mechanics and Rock Engineering, vol. 46, no. 1, pp. 189-198, 2013.

[10] Y. Li, Q. Wang, J. Chen, L. Xu, and S. Song, “K-means Algorithm Based on Particle Swarm Optimization for the Identification of Rock Discontinuity Sets," Rock Mechanics and Rock Engineering, vol. 48, no. 1, pp. 375-385, 2014.

[11] J. Liu, X.-D. Zhao, and Z.-H. Xu, "Identification of rock discontinuity sets based on a modified affinity propagation algorithm," International Journal of Rock Mechanics and Mining Sciences, vol. 94, pp. 32-42, 2017.

[12] J. Zhan, J. Chen, P. Xu, W. Zhang, X. Han, and X. Zhou, "Automatic identification of rock fracture sets using finite mixture models," Mathematical Geosciences, vol. 49, no. 8, pp. 1021-1056, 2017.

[13] M.-F. Cai, P. Wang, K. Zhao, and D.-K. Zhang, "Fuzzy Cmeans cluster analysis based on genetic algorithm for automatic identification of joint sets," Chinese Journal of Rock Mechanics and Engineering, vol. 24, no. 3, pp. 371-376, 2005.

[14] B. Lu, X. Ding, and A. Wu, "Study on method of orientation data partitioning of randomly distributed discontinuities of rocks," Chinese Journal of Rock Mechanics and Engineering, vol. 26, no. 9, pp. 1809-1816, 2007.

[15] Y.-X. Zhou, Z.-F. Zhou, and Q.-G. Sun, "Synthetical fuzzy clustering analysis for joints occurrence of rock mass," Chinese Journal of Rock Mechanics and Engineering, vol. 24, no. 13, pp. 2283-2287, 2005.
[16] S.-B. He and J. Liu, "Dominant Grouping of Rock Mass Structural Space Orientation Based on Spectral Clustering Algorithm," Gold Science and Technology, vol. 25, no. 4, pp. 4651, 2017.

[17] S. Song, Q. Wang, J. Chen, Y. Li, W. Zhang, and Y. Ruan, "Fuzzy $\mathrm{C}$-means clustering analysis based on quantum particle swarm optimization algorithm for the grouping of rock discontinuity sets," KSCE Journal of Civil Engineering, vol. 21, no. 4, pp. 11151122, 2017.

[18] Y.-R. Liu and H.-M. Tang, Rockmass Mechanics, China University of Geosciences Press, Wuhan, China, 1999.

[19] B. Tokhmechi, H. Memarian, B. Moshiri, V. Rasouli, and H. A. Noubari, "Investigating the validity of conventional joint set clustering methods," Engineering Geology, vol. 201, no. 3-4, pp. 75-81, 2005.

[20] W. Zhou and N. H. Maerz, "Implementation of multivariate clustering methods for characterizing discontinuities data from scanlines and oriented boreholes," Computers \& Geosciences, vol. 28, no. 7, pp. 827-839, 2002.

[21] L.-M. Xu, J.-P. Chen, and Q. Wang, "Study of method for multivariate parameter dominant partitioning of discontinuities of rock mass," Yantu Lixue/Rock and Soil Mechanics, vol. 34, no. 1, pp. 189-195, 2013.

[22] S.-Y. Song, Q. Wang, J.-P. Chen, Y.-Y. Li, and M.-Y. Shi, "A method for multivariate parameter dominant partitioning of discontinuities of rock masses," Yantu Lixue/Rock and Soil Mechanics, vol. 36, no. 7, pp. 2041-2048, 2015.

[23] Z.-Q. Bian and X.-G. Zhang, Pattern Recognition, Tsinghai University Press, Beijing, China, 2000.

[24] C.-Y. Song, Y.-Y. Zhang, and H.-D. Meng, "Research based on Euclid distance with weights of clustering method," Computer Engineering and Applications, vol. 04, pp. 179-180, 2007.

[25] J.-P. Chen, S.-F. Xiao, and Q. Wang, Stochastic discontinuity three-dimensional network computer simulation principle, Northeast Normal University Press, Changchun, China, 1995.

[26] J.-Y. Zeng, "Principle and implementation of ISODATA algorithm," Science Mosaic, vol. 07, pp. 126-127, 2009.

[27] T. Zhan and Y. Zhou, "Fuzzy partitional clustering algorithm," Journal of Software, vol. 6, pp. 858-868, 2004.

[28] L.-K. Meng and C.-C. Hu, "Cluster validity index based on measure of fuzzy partition," Journal of Electrical and Computer Engineering, vol. 11, pp. 15-17, 2007.

[29] F.-Y. Wang, Engineering application of rapid acquiring rock mass fractures information with digital close range photogrammetry, Jilin University, 2006.

[30] S. Song, Q. Wang, J. Chen, Y. Li, Q. Zhang, and C. Cao, "A multivariate method for identifying structural domain boundaries in a rock mass," Bulletin of Engineering Geology and the Environment, vol. 74, no. 4, pp. 1407-1418, 2015. 


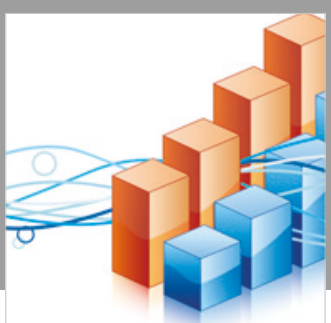

Advances in

Operations Research

\section{-n-m}
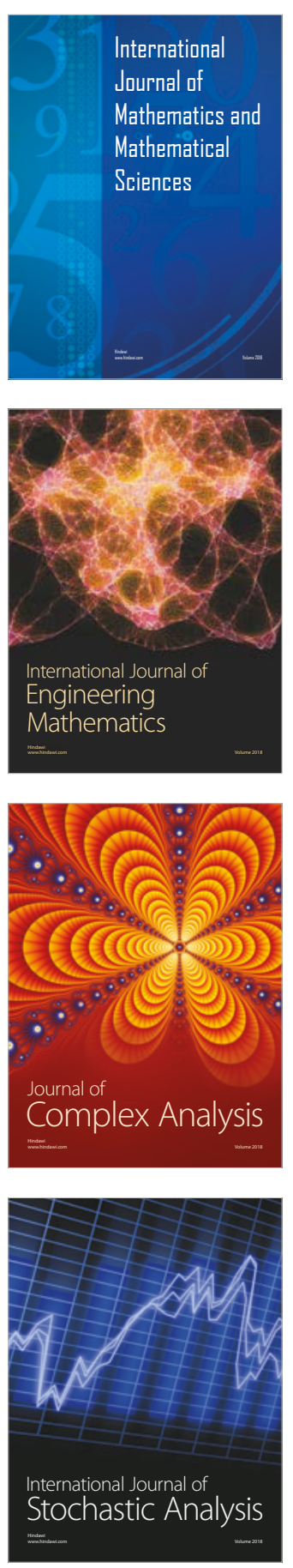
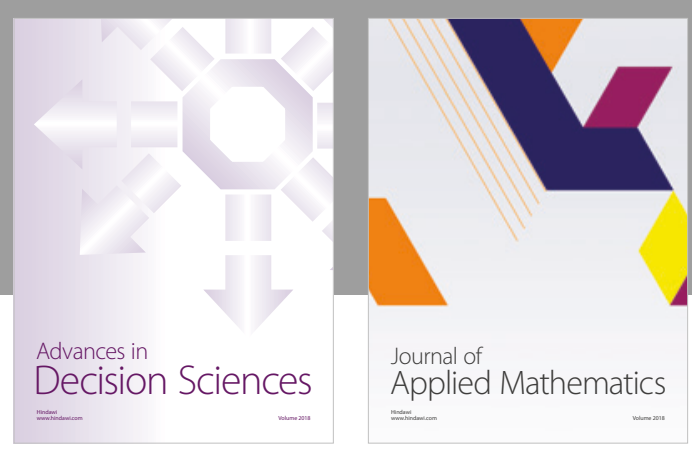

Journal of

Applied Mathematics
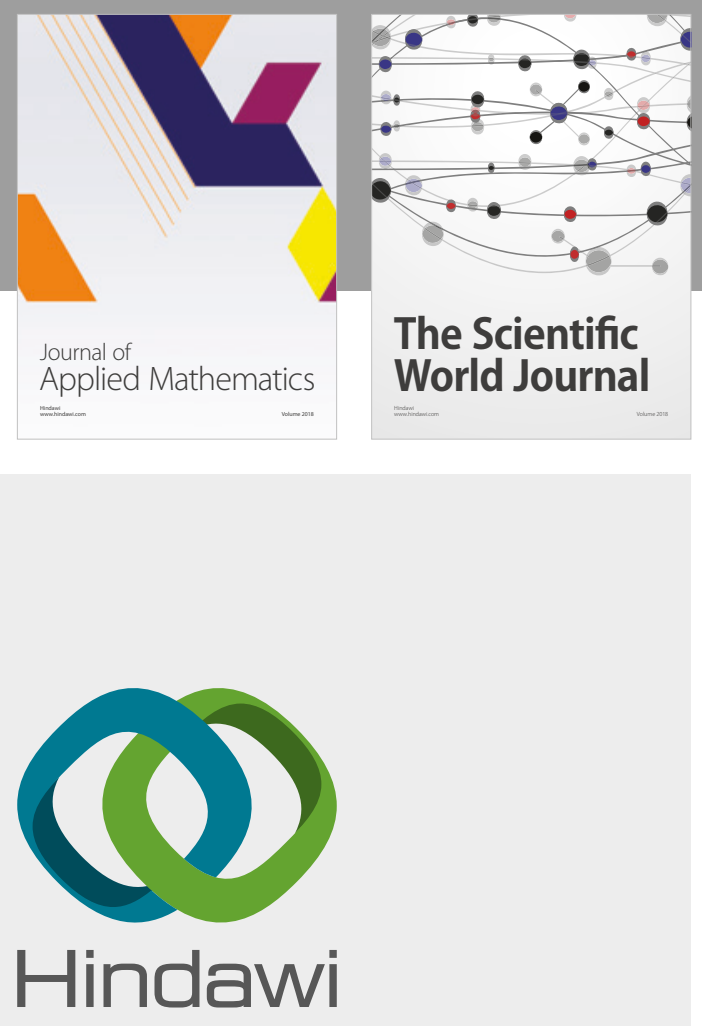

Submit your manuscripts at

www.hindawi.com

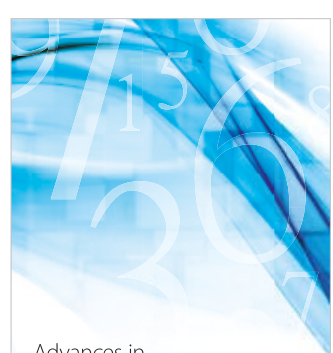

Advances in
Numerical Analysis
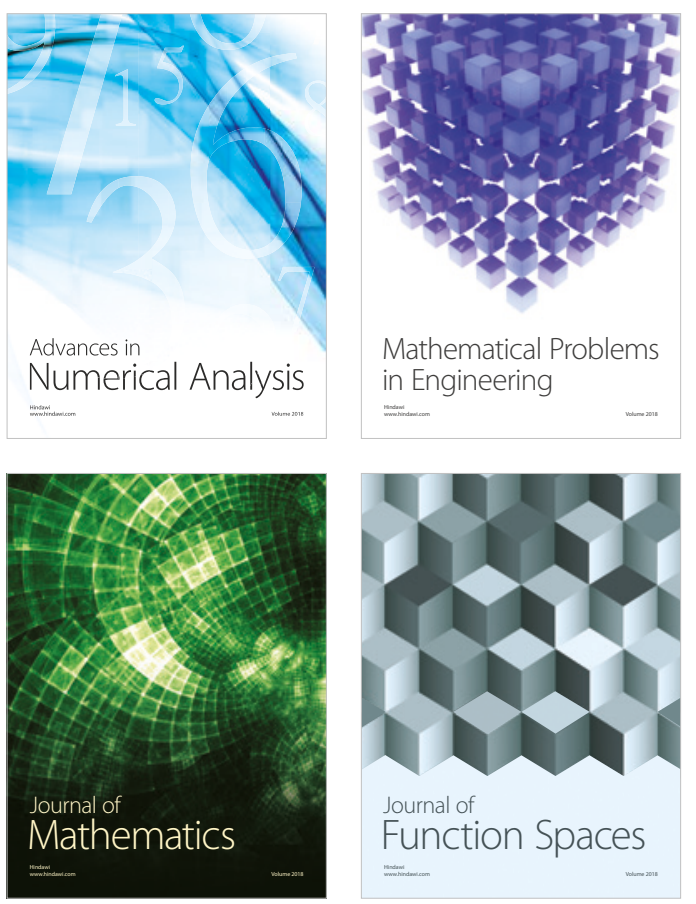

Mathematical Problems in Engineering

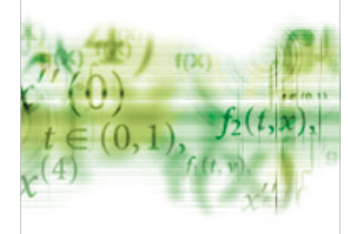

International Journal of

Differential Equations

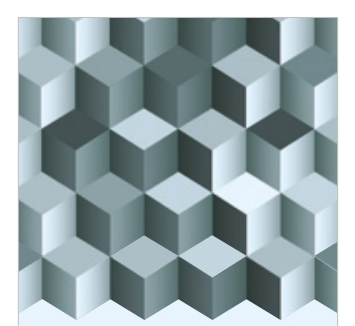

Journal of

Function Spaces

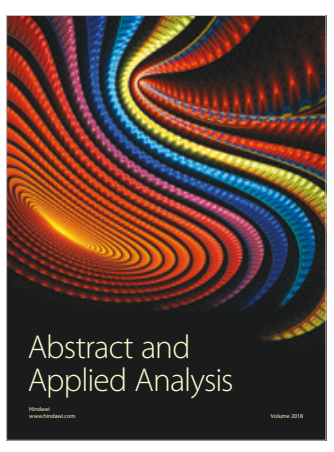

The Scientific

World Journal

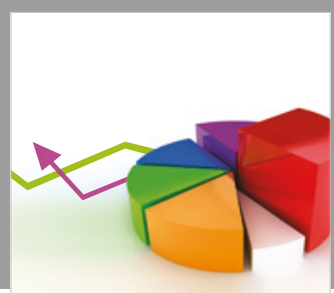

Journal of

Probability and Statistics
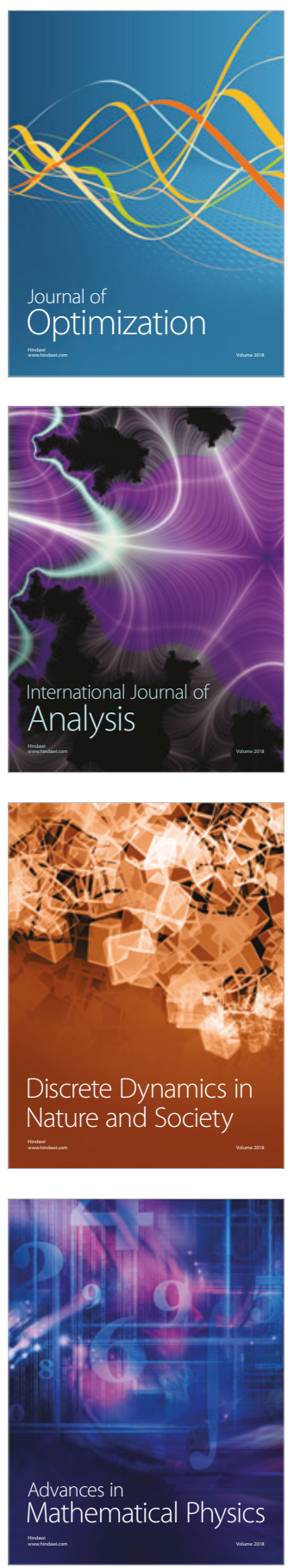